\title{
JSI TRIGA neutron and gamma field characterization by TLD measurements
}

\author{
$1^{\text {st }}$ Klemen Ambrožič \\ Reactor physics division
Jožef Stefen Institute \\ Ljubljana, Slovenia \\ klemen.ambrozic@ijs.si \\ $4^{\text {th }}$ Luka Snoj \\ Reactor physics division \\ Jožef Stefen Institute \\ Ljubljana, Slovenia \\ luka.snoj@ijs.si
}

\author{
$2^{\text {nd }}$ Klaudia Malik
}

Department of Radiation Physics and Dosimetry

Institute of Nuclear Physics

Krakow, Poland

klaudia.malik@ifj.edu.pl

\author{
$3^{\text {rd }}$ Barkara Obryk \\ Department of Radiation Physics and Dosimetry \\ Institute of Nuclear Physics \\ Krakow, Poland \\ barbara.obryk@ifj.edu.pl
}

\begin{abstract}
A well characterized radiation field inside a research nuclear reactor irradiation facilities enables precise qualification of radiation effects to the irradiated samples such as nuclear heating or changes in their electrical or material properties. To support the increased utilization of the JSI TRIGA reactor irradiation facilities in the past few years mainly on account of testing novel detector designs, electronic components and material samples, we are working on increasing the neutron and gamma field characterization accuracy using various modeling and measurement techniques.

In this paper we present the dose field measurements using thermo-luminescent detectors (TLD's) with different sensitivities neutron and gamma sensitivities, along with multiple ionization and fission chamber. Experiment was performed in several steps from reactor start-up, steady operation and a rapid shutdown, during which the ionization and fission chamber signals were acquires continuously, while the TLD's were being irradiated at different stages during reactor operation and after shutdown, to also capture response to delayed neutron and gamma field. The results presented in this paper serve for validation of JSI designed JSIR2S code for delayed radiation field determination, initial results of its application on the JSI TRIGA TLD measurements will also be presented.
\end{abstract}

Index Terms-TRIGA, MCP-N, MCP-7, TLD, ionization chamber, fission chamber, R2S, delayed gamma field

\section{INTRODUCTION}

In order to increase the utilization of irradiation facilities inside a research nuclear reactor for purpose of sample irradiation and characterization of its response, a detailed radiation field characterization is required. This is usually performed as a combination of experiments and simulations, which are also validated by experimental data.

The Jožef Stefan Institute (JSI) TRIGA Mark II research reactor is equipped with numerous in-core and ex-core irradiation facilities, utilization of which has increased mainly on account of a well characterized neutron field but also increasing accuracy of gamma field characterization for the novel radiation detectors and measurement techniques [1],

Identify applicable funding agency here. If none, delete this. radiation tolerance testing [2] and material sample irradiation [3].

Numerous activities for validation of the computational model such as reaction rate measurements [4], [5], neutron and gamma field measurements using fission and ionization chambers [6], [7] have led to a validated representative computational model, which has been used for a more rigorous characterization of a steady state radiation field inside its irradiation facilities [8], [9]. The next step is to capture temporally dependent radiation field behavior, especially gamma due to decay of activation and fission products, which amounts to up to $20 \%$ to $30 \%$ [10], depending on operational history and position inside the reactor core. The previous work on delayed radiation field characterization dealt with relative values in terms of detector responses of delayed gamma field vs. total gamma field. In order to obtain absolute dose-rate values, a series of TLD measurements were performed in different irradiation, which were irradiated during different stages of of reactor operation and after shutdown.

In this paper we present the use of nautroal and enriched Li TLD's for measurements of high intensity mixed radiation field of the JSI TRIGA reactor irradiation facilities, using high temperature readout techniques for a dose measurement range from 0.1 Gy to MGy.

\section{TLD DETECTORS}

Thermoluminescence detectors (TLD's) based on the LiF compounds are widely used for mixed field dosimetry. Novel, high temperature TLD readout at temperatures up to $600{ }^{\circ} \mathrm{C}$ has revealed an additional high temperature dosimetric peak, with the main dosimetric peak at $220^{\circ} \mathrm{C}$ disappearing with increasing doses. A new, high temperature dosimetric peak, called "peak B" and the UHTR (Ultra High Temperature Ratio) readout technique was developed at the IFJ enabling dose readouts ranging from $\mu \mathrm{Gy}$ to MGy [11]-[14].

In order to demonstrate the usefulness of MCP TLDs in a wide range of doses inside a mixed radiation field of a nuclear 
reactor, 50 dosimeter packets were prepared, each consisting of $3^{\text {nat }} \mathrm{LiF}: \mathrm{Mg}, \mathrm{Cu}, \mathrm{P}$ (MCP-N) TLDs, with ${ }^{6} \mathrm{Li}$ being highly sensitive to neutrons and $3{ }^{7} \mathrm{LiF}: M g, C u, P$ TLDs with enriched lithium 7 (MCP-7) manufactured by the IFJ PAN,Kraków, Poland (Fig. 1). The calibration to the neutron radiation field component to the TLD dose has been performed in the thermal neutron facility at INMRI laboratories in ENEA Casaccia [15].

The goal of using both MCP-N and MCP-7 TLD's was to distinguish neutron and non-neutron contributions to the dose and to evaluate the measurement range uncertainty.

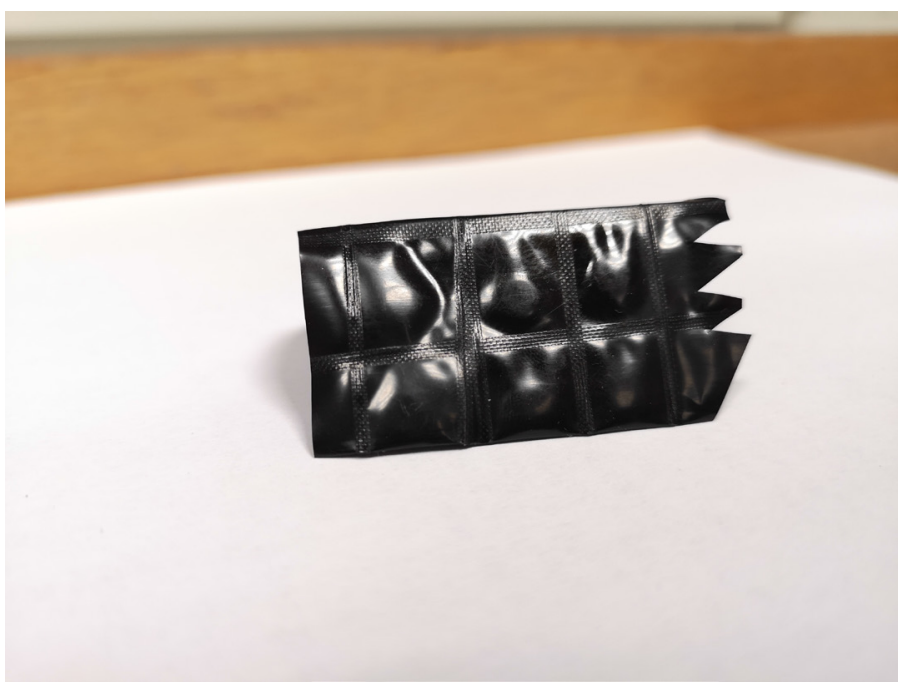

Fig. 1. 6 TLD pills, enclosed in a plastic foil packet.

\section{MeAsurements AT THE JSI TRIGA REACTOR}

\section{A. Description of the JSI TRIGA reactor}

The JSI TRIGA reactor is a $250 \mathrm{~kW}$ steady state power, pool type research reactor, which can also be operated in a pulse mode up to $2 \mathrm{GW}$ of thermal power. The reactor equipped with numerous irradiation facilities. The reactor core has 95 positions, 4 of which are filled with control rods, and the rest are filled by either fuel elements, irradiation channels or are left empty (Fig. 2). The Pulse control rod is used for reactor pulse operation, and can be ejected from the reactor core by a pneumatic system while the Regulating, Compensating and Safety control rods are used for steady state operation. The latter are comprised of a neutron absorber, followed by a fuel follower, which gets inserted into th reactor core upon control rod withdrawal.

Fuel elements are of cylindrical geometry, consisting of U$\mathrm{ZrH}$ fuel meat with $12 \mathrm{wt} \%$ of $20 \%$ enriched uranium, inner $\mathrm{Zr}$ rod, and upper and lower graphite reflector. The entire fuel assembly is clad in stainless steel.

In core irradiation channels consist of an aluminum tube, with or without a bottom graphite distance holder (Fig. 3). Some of the irradiation positions are equipped with a fast pneumatic transfer system.
The reactor core is surrounded by a graphite detector with a rotary carousel holding 40 irradiation positions (Fig. 2).

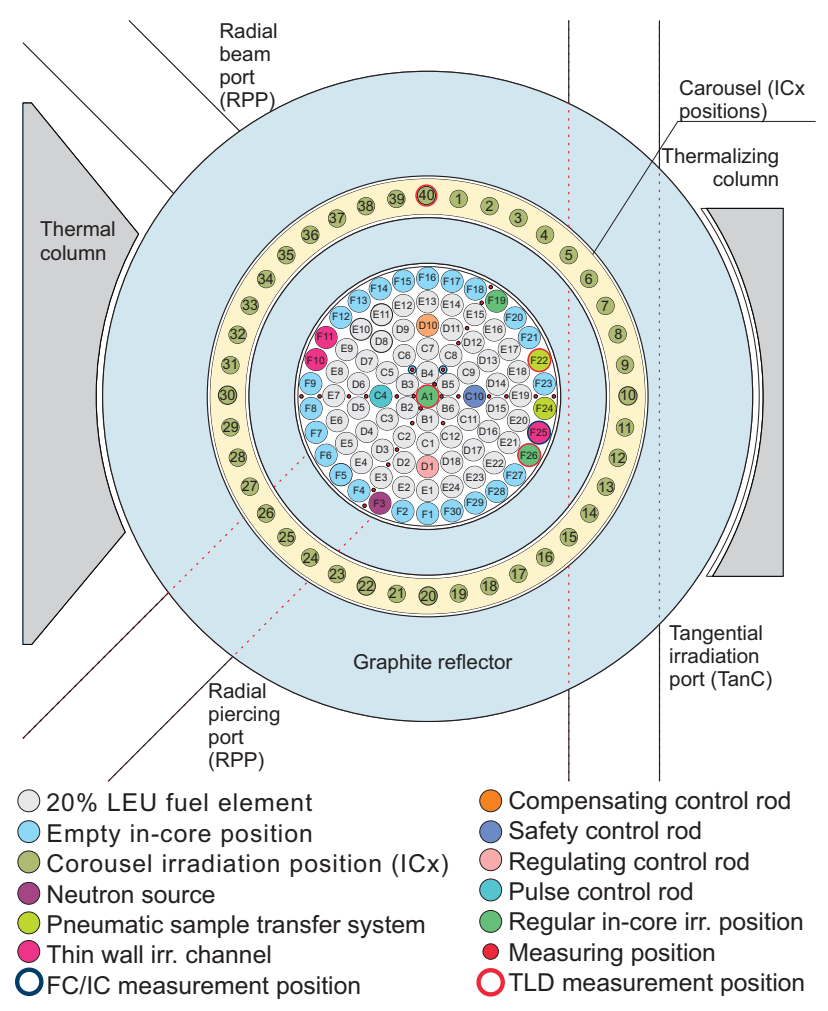

Fig. 2. JSI TRIGA core schematic, as arranged during the experiment.

\section{B. Pre-experimental preparation}

In order to prepare for the TLD irradiations, a comprehensive computational study of steady state neutron dose rate and prompt only gamma dose rate has been performed, to determine desired reactor power and irradiation time. Expected dose-rates were taken from [8]. For covering the desired measured dose range of $1 \mathrm{kGy}$ to $100 \mathrm{kGy}$ the $\mathrm{F} 22$ irradiation position, equipped with the pneumatic transfer system was selected and preliminary irradiations were performed.

One of the experimental goals was also to measure dose-rate dependence on the distance from the reactor core center and trying to distinguish prompt and delayed radiation contributions to the dose. 3 irradiation positions: Central channel, and irradiation channel in F26 in core position and IC40 position inside the graphite reflector with distinctively different doserates were selected. Since delayed radiation field depends on the operational history of the reactor, the experiment was to be performed in steps by reactor power-up to steady power, followed by a rapid shutdown, increasing the steady power of the step from $50 \mathrm{~W}$ to $100 \mathrm{~kW}$, in order to prevent decay radiation from the previous step to significantly influence the following one, with intermediate cooling times roughly twice the irradiation times.

At the end a series of TLD irradiations were to be performed at full reactor power, using the pneumatic transfer system in 


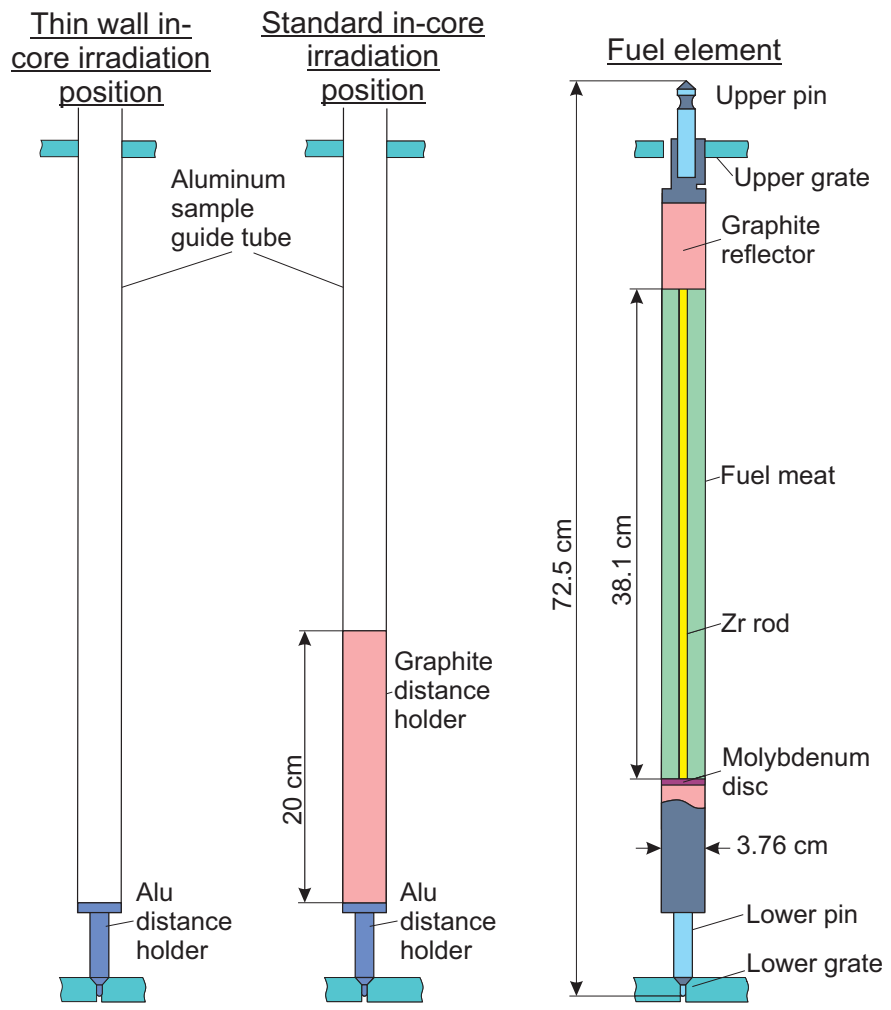

Fig. 3. JSI TRIGA fuel element and in-core irradiation positions schematics.

order not to introduce additional errors to the measurement time and not to increase the background.

\section{Experimental campaign}

The experimental campaign was executed in August 2017 along with synchronous measurements using multiple fission and ionization chambers [10] in order to measure and evaluate the relative temporal behavior of the gamma field and to provide continuous measurements of neutron flux and reactor power levels, which are used for comparison of measured TLD dose-rates and to provide data for modeling with JSIR2S code. For the first part, a series of reactor startup, operation at steady power level and rapid shutdown steps at steady reactor power ranging from $50 \mathrm{~W}$ to $5 \mathrm{~kW}$ were performed in increasing steady reactor power order. TLD packets inside the polyethylene rabbit containers were inserted into Central irradiation channel, F26 channel and IC40 position during steady reactor power and after shutdown (6 containers per power step).

One step at full reactor power was also performed, using 6 TLD packets during steady state operation, and 7 packets after reactor shutdown.

\section{EXPERIMENTAL RESULTS}

\section{A. Support of the experimental results}

During this experimental campaign, several ionization and a fission chamber have been inserted into the reactor core for neutron and gamma flux monitoring. These signals have been used for estimating the neutron and gamma contributions after reactor shutdown and for background estimations.

The prompt neutron and gamma dose values during reactor operation were taken from [8] and scaled to steady reactor power during each step accordingly, having a $5 \%$ uncertainty due to reactor power readout uncertainty. The delayed gamma has contribution has been estimated from [10] and scaled accordingly to the TLD irradiation positions, with relative uncertainty of $20 \%$ due to uncertainty of radial delayed gamma contribution dependence.

In between the steps, the background gamma radiation during the TLD irradiation was estimated from the calibrated PTW 30010 Farmer ionization chamber (PTW IC) located in the F25 position, and scaled accordingly for TLD irradiation positions by preliminary calculations of the delayed gamma contribution using the in-house developed JSIR2S code [16], where the uncertainty was estimated by the span of calculated dose ratios between PTW IC and the TLD positions at different times during an irradiation step respectively.The neutron dose in between the steps was determined by Miniature fission chamber measurements of neutron flux, located in MP17 position, and scaled accordingly to the specific TLD position and to the measured neutron flux from [8]. For MCP-N both neutron and gamma doses were summed, and only gamma doses attributed to the MCP-7 TLDs as described by [15].

This yielded a combined computational and experimental results, relying heavily on the previous work. For comparison with the TLD doses, the before mentioned will be regarded as computed dose values.

In the full paper, a detailed reproduction of the experiment using the Monte Carlo particle transport code MCNP [17] for prompt neutron and gamma dose rate calculations will be performed and a complete delayed radiation field evaluation using the JSIR2S code will be presented, and will serve as reference computed dose values.

\section{B. Results analysis}

In this section, TLD irradiation times are presented with respect to the neutron and gamma flux fluxes as measured by a fission and an ionization chamber respectively. In Figs. 4,5 and 6 the TLD insertion in their respective irradiation positions with respect to the ionization and fission chamber signals are presented. TLD irradiation time was set for $10 \mathrm{~min}$.

In Figs. 7, 8 and 9 the doses as measured by the MCP-7 and MCP-N are presented. The C/E values obtained, as described in the previous section are given on top of each bar, with the used TLD readout technique coded in $\mathrm{C} / \mathrm{E}$ color $(\mathrm{Ka}=1 \mathrm{~Gy}$ in red, UHTR(250) in blue and UHTR(350) in green). A trend can be observed depending on the readout technique, with $\mathrm{C} / \mathrm{E}$ TLD dose values obtained using $\mathrm{Ka}=1$ Gy ranging between 0.3 to 4 , for $\operatorname{UHTR}(250)$ ranging between 0.02 to 0.6 and in range of 0.06 to 0.1 for $\operatorname{UHTR}(350)$ readout techniques.

The MCP-N exhibit increased sensitivity to neutrons compared to MCP-7 during reactor operation, however this is not the case in between the reactor operation steps. The approximation that MCP-7 are sensitive only to gamma rays has been validated at low dose rates (mGy) [15]. However at dose rates in 
order of $i 100 \mathrm{~Gy} \mathrm{~h}^{-1}$ this does not appear to be the case. One of possible culprits for such discrepancy might be self heating by incident neutron via reactions on $\mathrm{Li}$ isotopes such as ${ }^{6} \mathrm{Li}+n \rightarrow{ }^{4} \mathrm{He}+{ }^{3} \mathrm{H}+4.78 \mathrm{MeV}$, which might cause self-annealing. Additional measurements, using MCP-N and MCP-7 TLD's in conjunction with a small thermo-couple are planned in the near future.

The measured dose-rates also differ significantly to the ones predicted by above mentioned efforts for support of experimental results. However different $\mathrm{C} / \mathrm{E}$ ranges can be observed, depending on the used readout technique. In order to identify possible reasons for large deviations of $\mathrm{C} / \mathrm{E}$ values from 1 the following activities will be performed in the near future:

- Temperature measurements of the TLD's during reactor operation have to be performed in order to rule out selfannealing of the dosimeters.

- Other dose-rate related effects, which might lead to response non-linearity.

- Sensitivity of both MCP-N and MCP-7 to neutron component of the radiation field.

- Enclosure in order to obtain charged particle equilibrium (CPE) for incident neutrons and gamma rays.

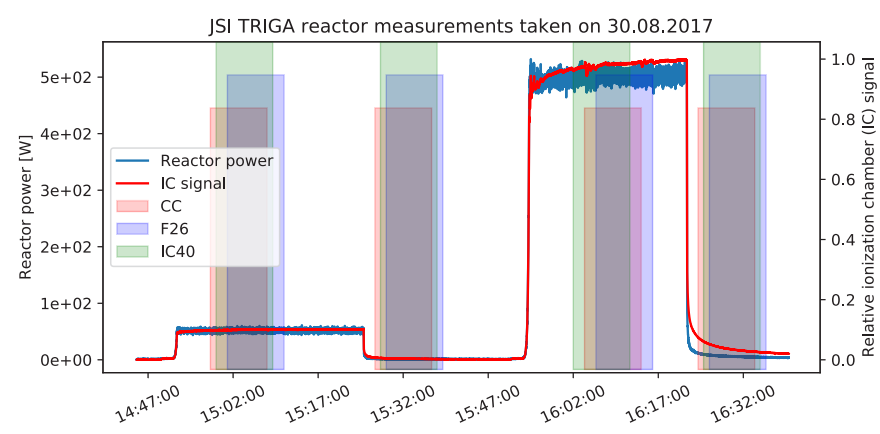

Fig. 4. Two irradiation step with steady power of $50 \mathrm{~W}$ and $500 \mathrm{~W}$.

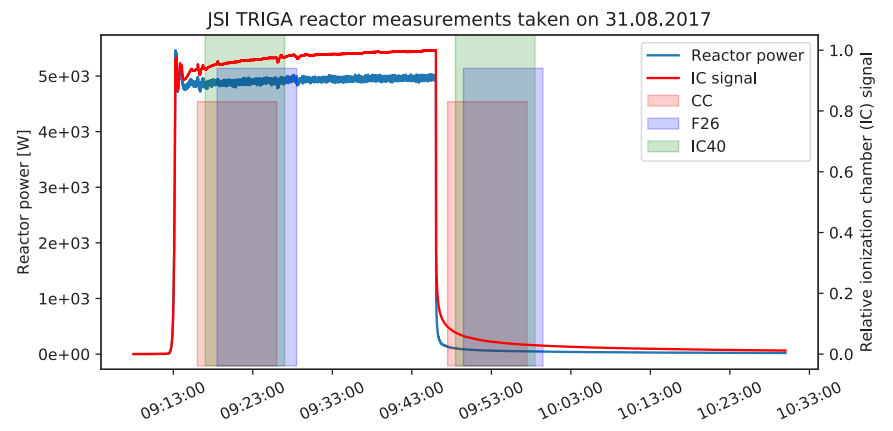

Fig. 5. Irradiation step with steady power of $5 \mathrm{~kW}$.

\section{CONCLUSiON}

Measurements using MCP-N with natural lithium and MCP7 with ${ }^{7} \mathrm{Li}$ isotope have been performed in an intense mixed radiation field of the JSI TRIGA reactor irradiation facilities

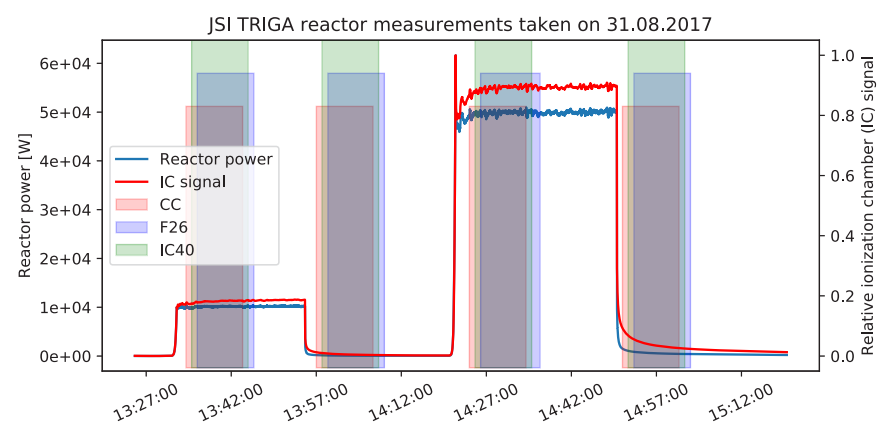

Fig. 6. Two irradiation steps with steady power of $10 \mathrm{~kW}$ and $50 \mathrm{~kW}$.

at different reactor power levels for a fixed time duration of $600 \mathrm{~s}$.

A parallel analysis using a combination ionization and fission chamber detectors and previous experimental and computational results has been performed in order to compare to the measured doses by the TLDs. A full computational analysis of the TLD doses using the MCNP and JSIR2S code will be presented in the full paper and validated by ionization chamber measurement results.

Additional work is required in improvement of before mentioned TLD dose readout techniques, which can be grouped according to the presented $\mathrm{C} / \mathrm{E}$ values, however none of them close to 1 for both MCP-N and MCP-7 type TLD.

The simple methodology for discriminating the neutron and gamma dose by subtracting doses from MCP-N and MCP7, validated at low dose rates appears to yield ambiguous (negative) neutron dose results with large uncertainties. We postulate this effect might be due to heating of the TLDs due to neutron reactions on ${ }^{6} \mathrm{Li}$, causing self-annealing. Temperature measurements of the TLDs during irradiation inside the JSI TRIGA reactor using a thermocouple will be performed in the near future, and presented in the full paper. TLD readout dose dependence on dose-rate (especially neutron) and influence of ${ }^{6} \mathrm{Li}$ impurities in MCP-7 TLDs should be studied in the future.

Several issues in using the MCP-N and MCP-7 dosimeters in the high dose rate mixed radiation field environment have been identified with proposed steps for their qualification or resolution outlined.

\section{REFERENCES}

[1] L. Barbot, V. Radulovic, D. Fourmentel, L. Snoj, M. Tarchalski, V. Dewynter-Marty, and F. Malouch, "Calculation to experiment comparison of spnd signals in various nuclear reactor environments," in 2015 4th International Conference on Advancements in Nuclear Instrumentation Measurement Methods and their Applications (ANIMMA), Aprill 2015, pp. 1-7.

[2] G. Kramberger, V. Cindro, I. Mandić, M. Mikuž, and M. Zavrtanik, "Determination of detrapping times in semiconductor detectors," Journal of Instrumentation, vol. 7, no. 04, pp. P04 006-P04 006, apr 2012. [Online]. Available: https://doi.org/10.1088\\%2F1748-0221 $\% 2 \mathrm{~F} 7 \backslash \% 2 \mathrm{~F} 04 \backslash \% 2 \mathrm{Fp} 04006$

[3] E. Huseynov, A. Garibov, and R. Mehdiyeva, "Influence of neutron irradiation and temperature on the electric conductivity of sio2 nanoparticles," Journal of Electrostatics, vol. 74, pp. 73 - 78, 


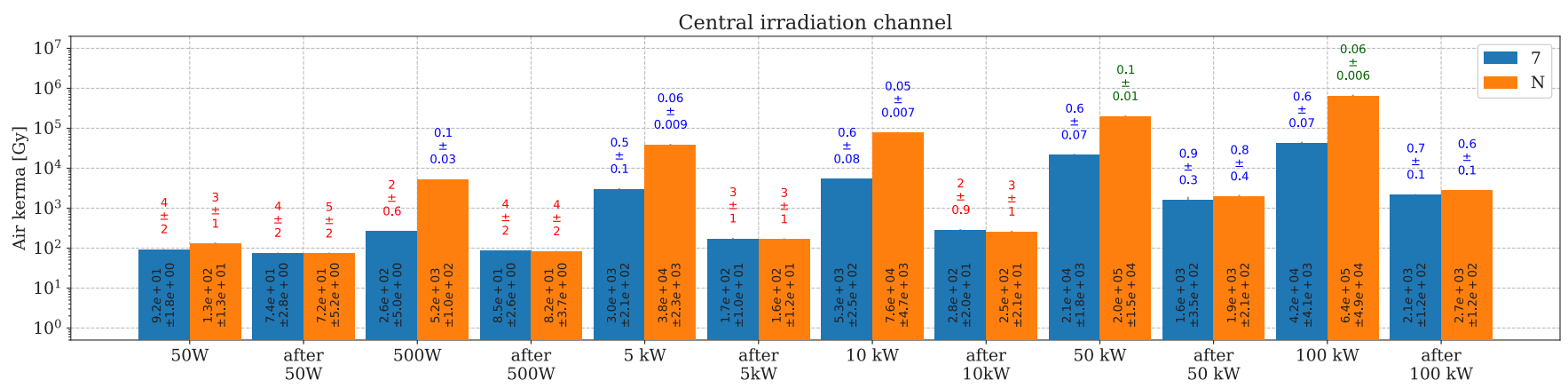

Fig. 7. Doses of TLD's irradiated inside the Central irradiation channel, with measured doses inside bars of the chart, and C/E values and their uncertainties in red above the bar charts.

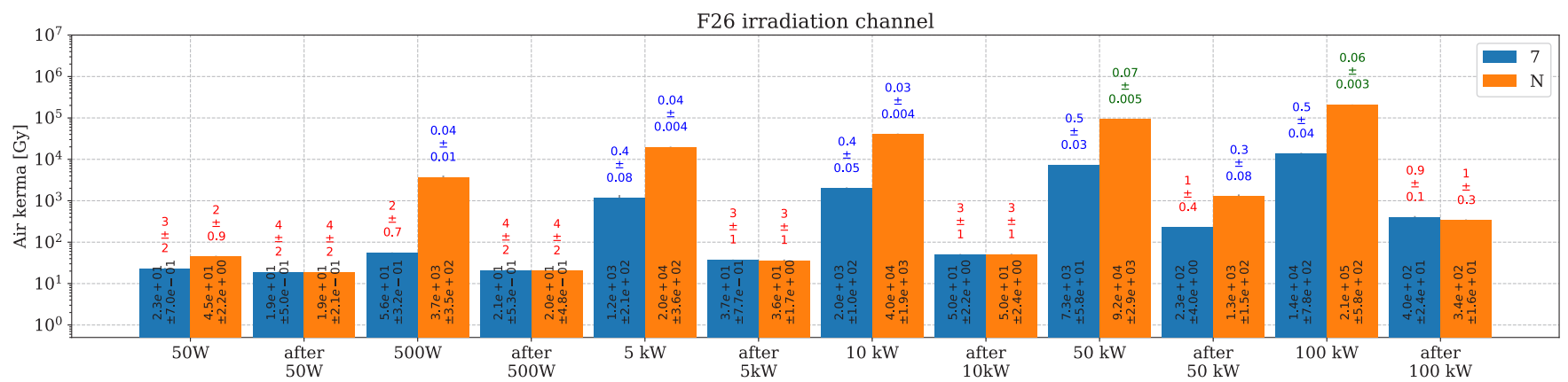

Fig. 8. Doses of TLD's irradiated inside the F26 irradiation channel, with measured doses inside bars of the chart, and C/E values and their uncertainties in red above the bar charts.

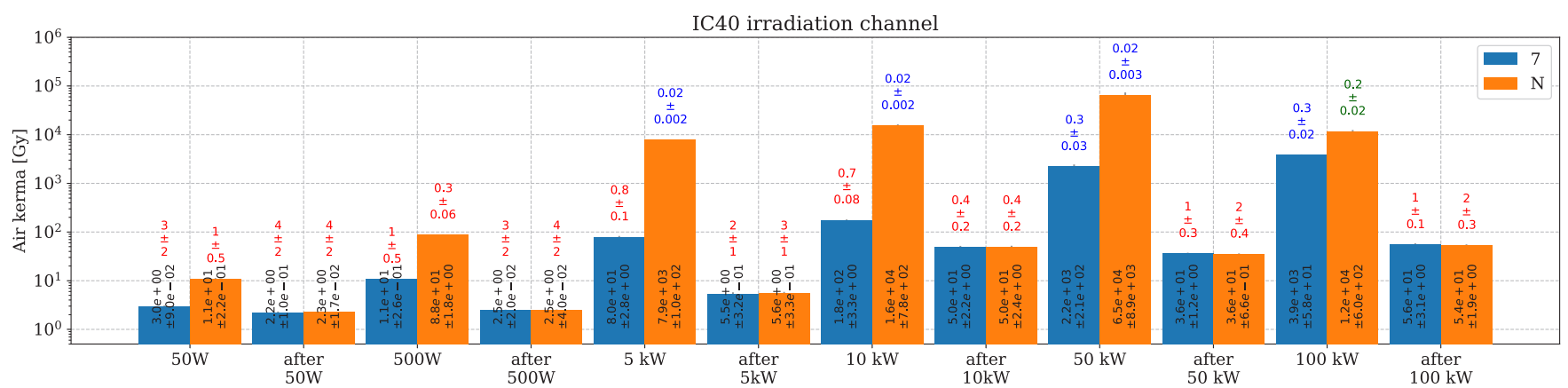

Fig. 9. Doses of TLD's irradiated inside the IC40 irradiation channel, with measured doses inside bars of the chart, and C/E values and their uncertainties in red above the bar charts.

2015. [Online]. Available: http://www.sciencedirect.com/science/article/ pii/S0304388615000029

[4] L. Snoj, A. Trkov, R. Jaćimović, P. Rogan, G. Žerovnik, and M. Ravnik, "Analysis of neutron flux distribution for the validation of computational methods for the optimization of research reactor utilization," Applied Radiation and Isotopes, vol. 69, no. 1, pp. 136 141, 2011. [Online]. Available: http://www.sciencedirect.com/science/ article/pii/S0969804310003611

[5] Štancar, Žiga, Kaiba, Tanja, Snoj, Luka, Barbot, Loïc, Destouches, Christophe, Fourmentel, Damien, and Villard, Jean-François, "Reaction rate benchmark experiments with miniature fission chambers at the slovenian triga mark ii reactor," EPJ Web Conf., vol. 170, p. 04023, 2018. [Online]. Available: https://doi.org/10.1051/epjconf/201817004023

[6] L. Barbot, C. Domergue, S. Bréaud, C. Destouches, J. Villard, L. Snoj, Ž. Stancar, V. Radulović, and A. Trkov, "Neutron field characterization of irradiation locations applied to the slovenian triga reactor," in 2013 3rd International Conference on Advancements in Nuclear Instrumentation, Measurement Methods and their Applications (ANIMMA), June 2013, pp. 1-5.

[7] G. Žerovnik, T. Kaiba, V. Radulović, A. Jazbec, S. Rupnik, L. Barbot, D. Fourmentel, and L. Snoj, "Validation of the neutron and gamma fields in the jsi triga reactor using incore fission and ionization chambers," Applied Radiation and Isotopes, vol. 96, pp. 27 - 35, 2015. [Online]. Available: http: //www.sciencedirect.com/science/article/pii/S0969804314003819

[8] K. Ambrožič, G. Žerovnik, and L. Snoj, "Computational analysis of the dose rates at jsi triga reactor irradiation facilities," Applied Radiation and Isotopes, vol. 130, pp. 140 - 152, 2017. [Online]. Available: http://www.sciencedirect.com/science/article/pii/S0969804317304633

[9] L. Snoj, G. Žerovnik, and A. Trkov, "Computational analysis of 
irradiation facilities at the jsi triga reactor," Applied Radiation and Isotopes, vol. 70, no. 3, pp. 483 - 488, 2012. [Online]. Available: http://www.sciencedirect.com/science/article/pii/S0969804311005963

[10] K. Ambrožič, A. Gruel, V. Radulović, M. L. Guillou, P. Blaise, C. Destouches, and L. Snoj, "Delayed gamma determination at the jsi triga reactor by synchronous measurements with fission and ionization chambers," Nuclear Instruments and Methods in Physics Research Section A: Accelerators, Spectrometers, Detectors and Associated Equipment, vol. 911, pp. 94 - 103, 2018. [Online]. Available: http://www.sciencedirect.com/science/article/pii/S0168900218312567

[11] P. Bilski, B. Obryk, P. Olko, E. Mandowska, A. Mandowski, and J. Kim, "Characteristics of lif:mg,cu,p thermoluminescence at ultra-high dose range," Radiation Measurements, vol. 43, no. 2, pp. 315 - 318, 2008, proceedings of the 15th Solid State Dosimetry (SSD15). [Online]. Available: http://www.sciencedirect.com/science/ article/pii/S1350448707004076

[12] B. Obryk, P. Bilski, M. Budzanowski, M. Fuerstner, M. Glaser, C. J Ilgner, P. Olko, A. Pajor, and Z. Stuglik, "Development of a method for passive measurement of radiation doses at ultra-high dose range," IEEE Transactions on Nuclear Science, vol. 56, no. 6, pp. 3759-3763, Dec 2009.

[13] B. Obryk, M. Glaser, I. Mandić, P. Bilski, P. Olko, and A. Sas-Bieniarz, "Response of various types of lithium fluoride mcp detectors to high and ultra-high thermal neutron doses," Radiation Measurements, vol. 46, no. 12, pp. 1882 - 1885, 2011, proceedings of the 16th Solid State Dosimetry Conference, September 19-24, Sydney, Australia. [Online]. Available: http://www.sciencedirect.com/science/ article/pii/S1350448711003088

[14] P. Bilski, B. Obryk, and Z. Stuglik, "Behaviour of lif:mg,cu,p and lif:mg,ti thermoluminescent detectors for electron doses up to 1mgy," Radiation Measurements, vol. 45, no. 3, pp. 576 - 578, 2010, proceedings of the 7th European Conference on Luminescent Detectors and Transformers of Ionizing Radiation (LUMDETR 2009 ). [Online]. Available: http://www.sciencedirect.com/science/article/pii/ S1350448709003679

[15] B. Obryk, R. Villari, P. Batistoni, A. Colangeli, P. D. Felice, N. Fonnesu, M. Kłosowski, S. Loreti, K. Malik, J. Nash, M. Pillon, M. Pimpinella, and L. Quintieri, "Tld calibration for neutron fluence measurements at jet fusion facility," Nuclear Instruments and Methods in Physics Research Section A: Accelerators, Spectrometers, Detectors and Associated Equipment, vol. 904, pp. 202 - 213, 2018. [Online]. Available: http://www.sciencedirect.com/science/article/pii/S0168900218308854

[16] K. Ambrozič, B. Kos, and L. Snoj, "Jsir2s code system for delayed radiation field calculations," in 2018 27th International Conference Nuclear Energy for New Europe (NENE), September 2018.

[17] J. T. Goorley, M. R. James, T. E. Booth, F. B. Brown, J. S. Bull, L. J. Cox, J. W. J. Durkee, J. S. Elson, M. L. Fensin, R. A. I Forster, and et al., Initial MCNP6 Release Overview - MCNP6 version 1.0. Los Alamos National Laboratory (LANL), Jun 2013, Report number: LANL Report LA-UR-13-22934. [Online]. Available: http://www.osti.gov/scitech/servlets/purl/1086758 